REVIEW ARTICLE

\title{
Italian neonatologists and SARS-CoV-2: lessons learned to face coming new waves
}

\author{
Maria Elena Cavicchiolo (D) ${ }^{1}$, Daniele Trevisanuto ${ }^{1}$, Elena Priante ${ }^{1}$, Laura Moschino ${ }^{1}$, Fabio Mosca ${ }^{2}$ and Eugenio Baraldi ${ }^{1,3}$
}

The aim of this review was threefold: (a) to retrieve all SARS-CoV-2 evidences published by Italian neonatologists working in maternity centers and NICUs during the pandemic; (b) to summarize current evidence for the management of term and preterm infants with a SARS-CoV-2-related illness; and (c) to provide an update for dealing with the second wave of COVID-19 and discuss open questions. A review was conducted using MEDLINE/PubMed and the national COVID-19 registry of the Italian Society of Neonatology including citations from December 1, 2019 to October 28, 2020. Sixty-three articles were included. Collected data were divided into the following topics: (a) antenatal management, (b) management in delivery room, (c) postnatal management, (d) mother-baby dyad and breastfeeding management, (e) neonatal emergency transport system reorganization, (f) parents' management and perspective during SARS-CoV-2 pandemic, and (g) future perspective. Evidences have evolved over the pandemic period and the current review can be useful in the management of the mother-neonate dyad during SARS-CoV-2 future waves. Italian neonatologists have played an active role in producing official guidelines and reporting data that have contributed to improve the care of neonates. A joint European action plan is mandatory to face COVID-19 in neonates with more awareness.

Pediatric Research (2022) 91:513-521; https://doi.org/10.1038/s41390-021-01477-8

\section{IMPACT:}

- A joint European action plan is mandatory to face COVID-19 in neonates with more awareness.

- This review summarizes the available evidences from neonatal COVID-19 management in Italy analyzing all the published paper in this specific field of interest.

- The current review can be useful in the management of the mother-neonate dyad during the SARS-CoV-2 future waves.

\section{INTRODUCTION}

Italy was one of the most exposed countries to pandemic severe acute respiratory syndrome coronavirus 2 (SARS-CoV-2) leading to a coronavirus disease 2019 (COVID-19) in >600,000 cases and 38,000 deaths.'

A profound reorganization of the clinical management of patients was necessary to address the SARS-CoV-2 health emergency throughout different levels of care. In the absence of evidence-based guidelines, physicians initially had to rely only on case reports/series and expert opinions for the management of these patients. This uncertainty has led to a little consistency in the management of similar patients in similar settings.

COVID-19 seems a mild disease in children, but neonates and infants $<1$ year appear to be at higher risk of severe respiratory failure than initially thought ${ }^{2}$ and the exact epidemiology and mechanisms are still under investigation. ${ }^{3,4}$

For these reasons, the Italian Society of Neonatology (SIN) and the main Italian neonatal intensive care units (NICUs) have issued documents aimed to offering updated recommendation on the management of the maternal-infant dyad, the reorganization of the maternal wards, the NICUs and its services, and to report and summarize the positive cases. ${ }^{5,6}$

The objective of the present review was to retrieve all SARSCoV-2 evidences published by neonatologists working in maternity centers and NICUs in Italy during the pandemic with the aim to summarize current evidence and open questions for the management of term and preterm infants with a SARS-CoV-2related illness in NICU, maternal ward, and staff reorganization and to provide an update and guide for dealing with the second wave of COVID-19.

\section{METHODS}

A review was conducted according to the Preferred Reporting Items for Systematic Reviews and Meta-Analyses guidelines. ${ }^{7}$ We conducted this research using MEDLINE/PubMed as international database including citations from December 1, 2019 to October 28, 2020, with the following search strategy of free-text terms: "coronavirus" OR "sars-cov-2" OR "covid-19" AND "Italy" AND "newborn" OR "preterm infant" OR "premature" AND "NICU". We also searched the national COVID-19 registry of the SIN available at: https://www.sin-neonatologia.it/covid-19/.

\section{Inclusion criteria}

Studies were eligible for full-text review if they included at least one of the co-authors affiliated to an Italian maternal ward or NICU. Retrospective studies, reviews, clinical or expert opinion, case report, or series were included. No language restrictions were applied.

\footnotetext{
'Department of Women's and Children's Health, Neonatal Intensive Care Unit, University Hospital of Padua, Padua, Italy; ${ }^{2}$ Neonatal Intensive Care Unit, Fondazione IRCCS Ca' Granda Ospedale Maggiore Policlinico, University of Milan, Milan, Italy and ${ }^{3}$ Institute of Pediatric Research (IRP), Fondazione Città della Speranza, Padova, Italy Correspondence: Eugenio Baraldi (eugenio.baraldi@unipd.it)
} 


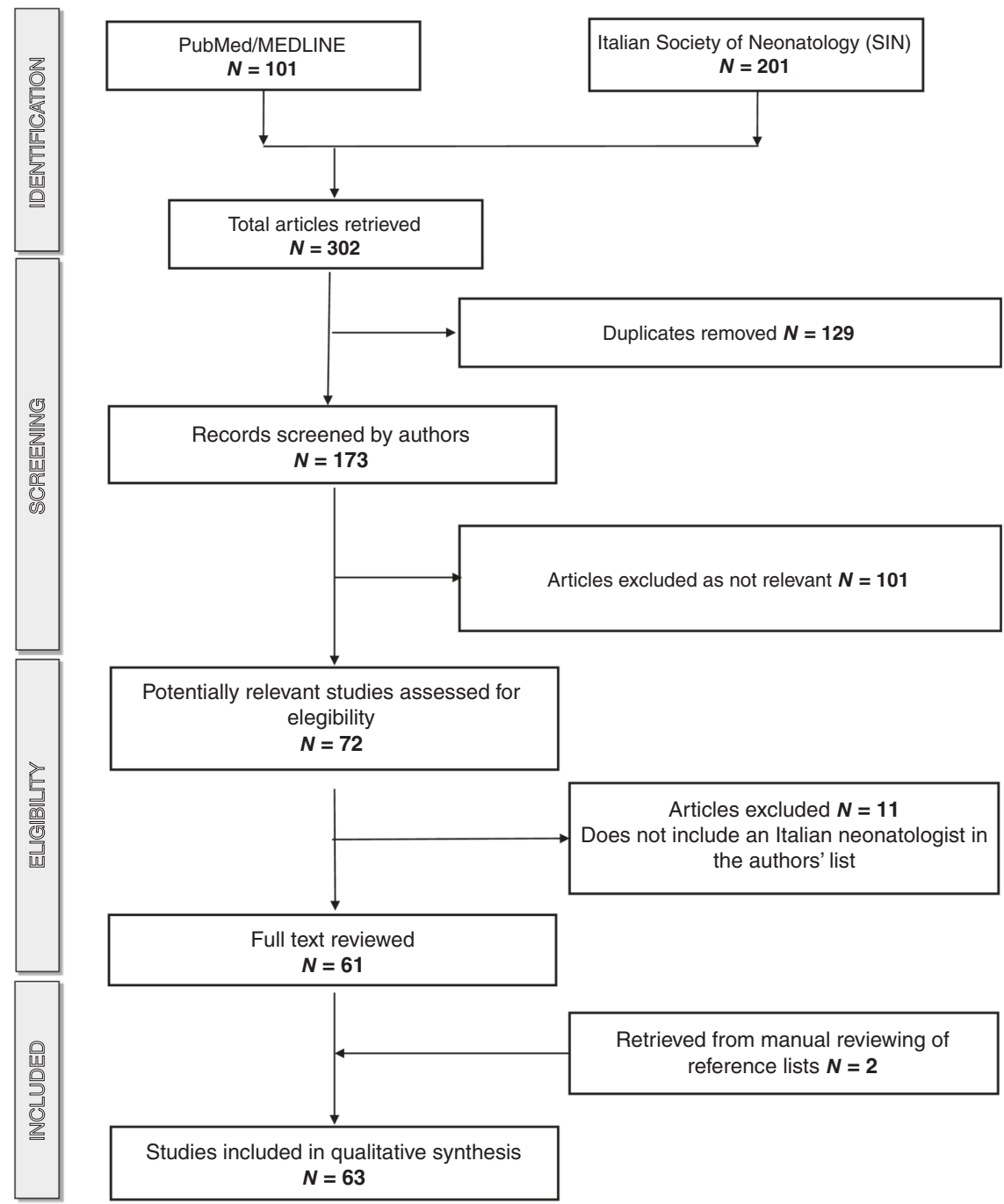

Fig. 1 Flow chart of the study selection process.

Study selection

Assessments of the titles, abstracts, and full texts were conducted independently by two investigators (M.E.C. and D.T.). Any disagreement regarding study selection was resolved by discussion with a third reviewer (E.B.). The selection process is summarized in Fig. 1.

Data collection

Data were extracted using a standardized data collection form, which summarized information about authors, author's center, year and month of publication, study design, topic, and international collaboration.

Data synthesis

We divided the collected data into the following topics: (a) antenatal management, (b) management in delivery room, (c) postnatal management, (d) Mother-baby dyad and breastfeeding management, (e) neonatal emergency transport system (NETS) reorganization, ( $f$ ) parents' management and perspective during SARS-CoV-2 pandemic, and (g) future perspective.

Statistical analysis

Since the study designs did not allow carrying out a meaningful meta-analysis, we performed a narrative synthesis of the included studies.

\section{RESULTS}

Study selection

Overall, the searches yielded 173 non-duplicated articles. Of them, 101 articles were excluded as not relevant. Eleven studies were furtherly excluded after authors' affiliation screening, and two additional studies were retrieved via hand search. Ultimately, 63 studies were included in the qualitative synthesis ${ }^{4,5,8-69}$ (Fig. 1). All articles were in English. Publication date was from March to October 2020. Sixteen out of 63 were expert or clinical opinion, 16 were reviews or systematic reviews, 15 were observational studies, 12 articles were case reports or series, and 14 were international collaborations (Table 1).

Antenatal management

Regarding the pre-triage to pregnant women, there was a change in practice during the first months of the pandemic. At the beginning, most maternity centers performed the pre-triage to pregnant women based on temperature and a specific questionnaire before entering the delivery room in order to identify as soon as possible suspected cases. These patients underwent nasopharyngeal swabs and were admitted to isolated facilities or transferred as suspected positives to designated maternity hubs. ${ }^{37}$ As the months went by, the increasing evidence suggested to perform universal screening with nasopharyngeal swabs to all pregnant women; this practice is now recognized in almost all 


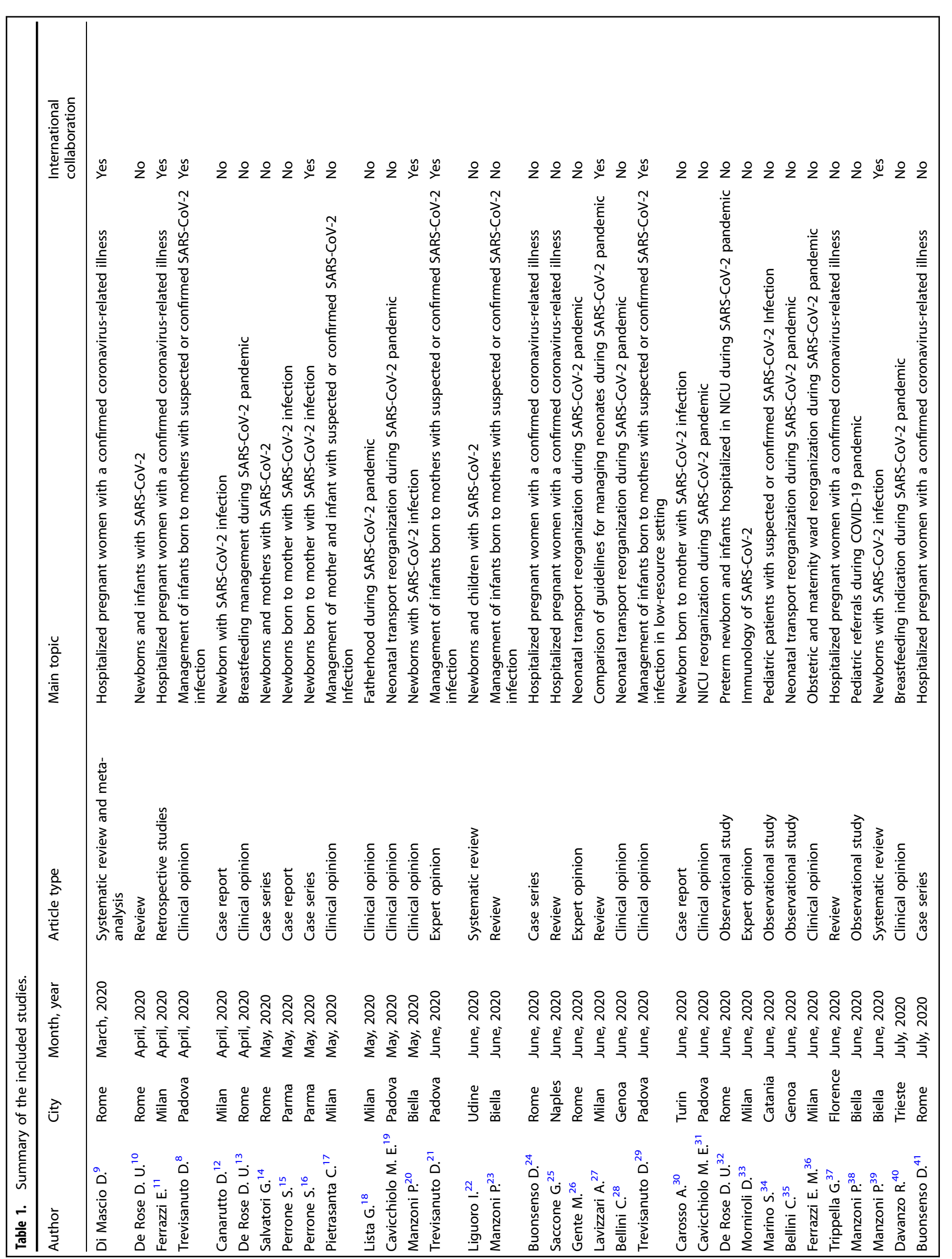

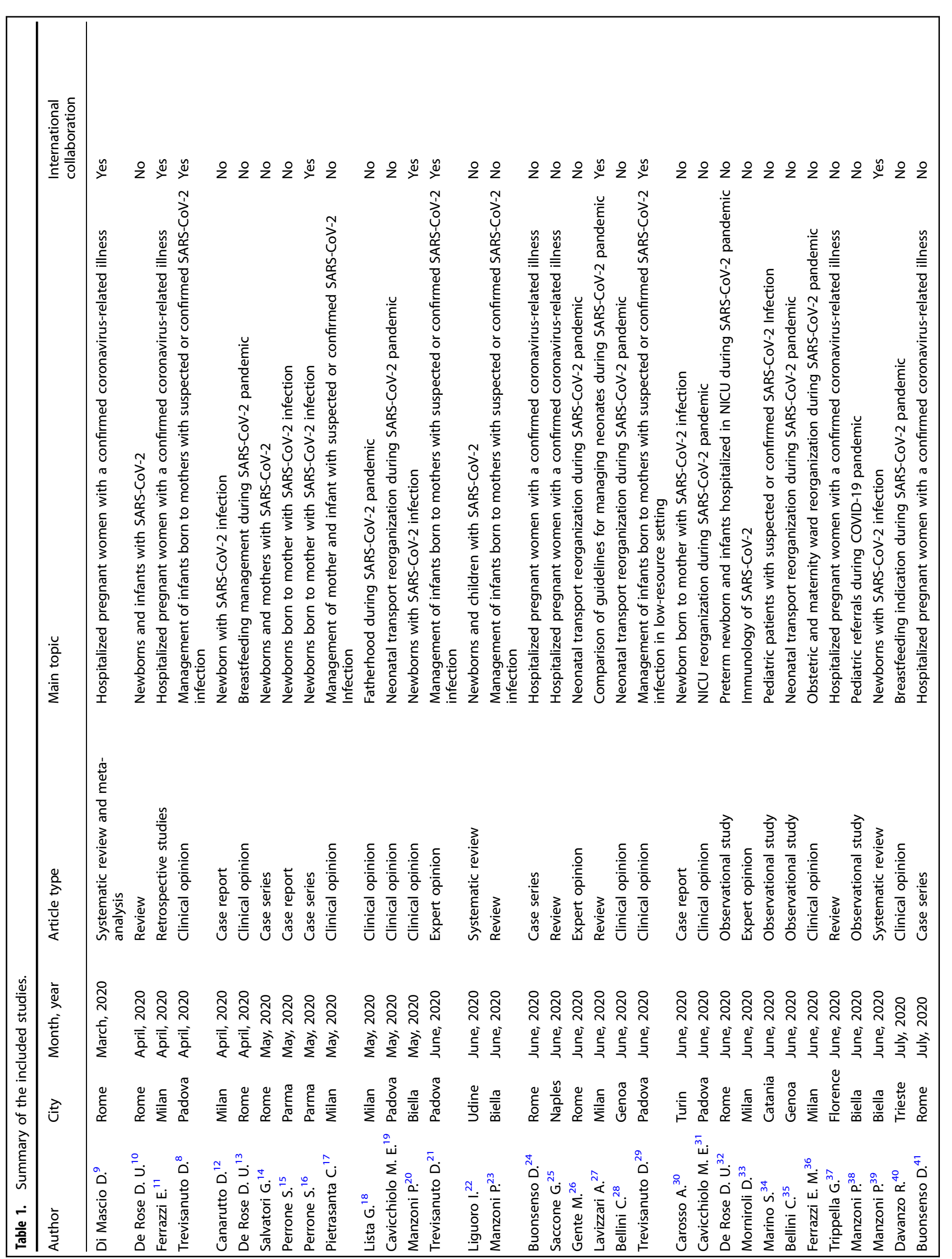

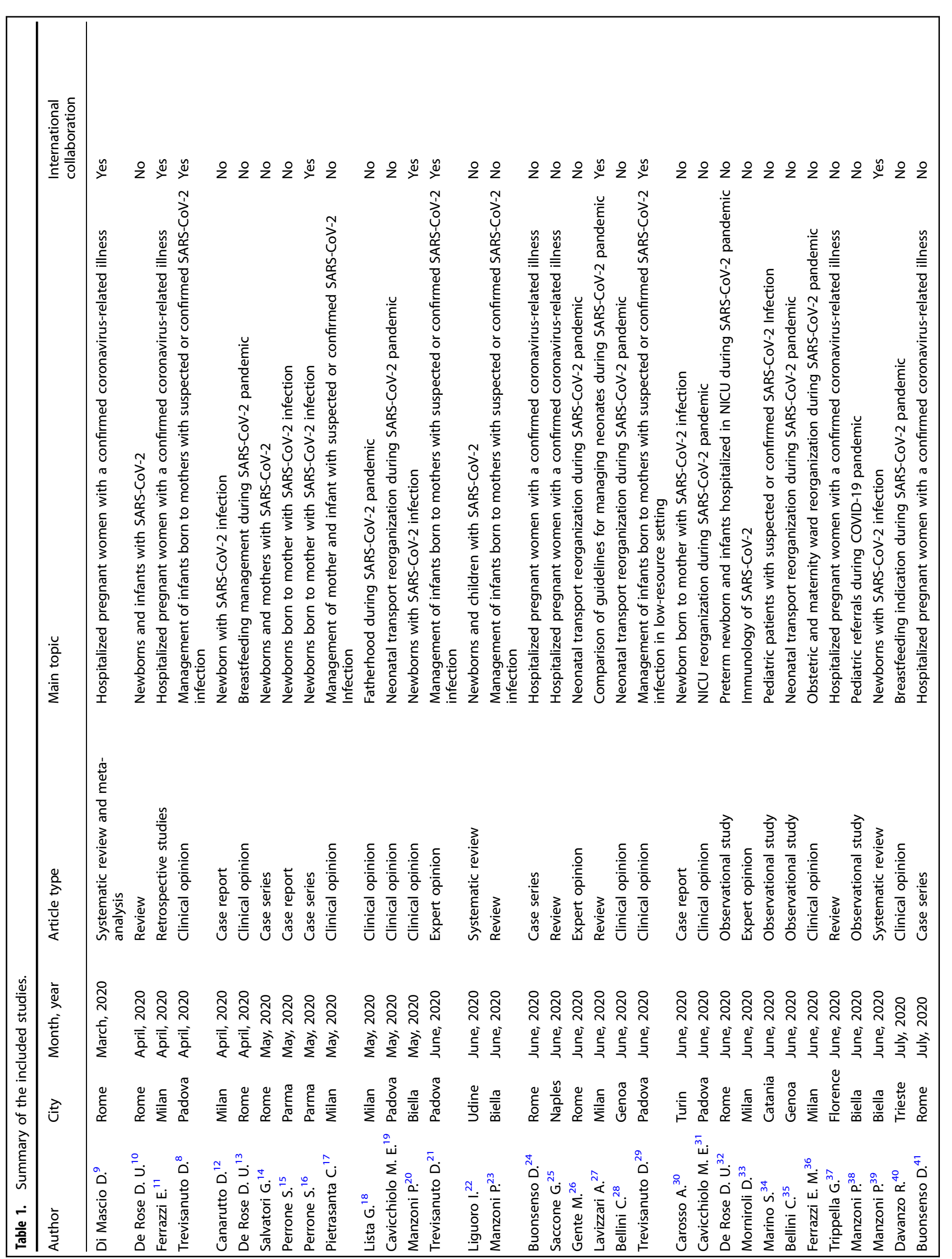




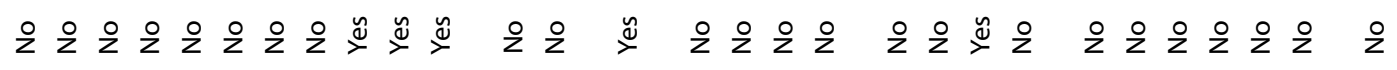

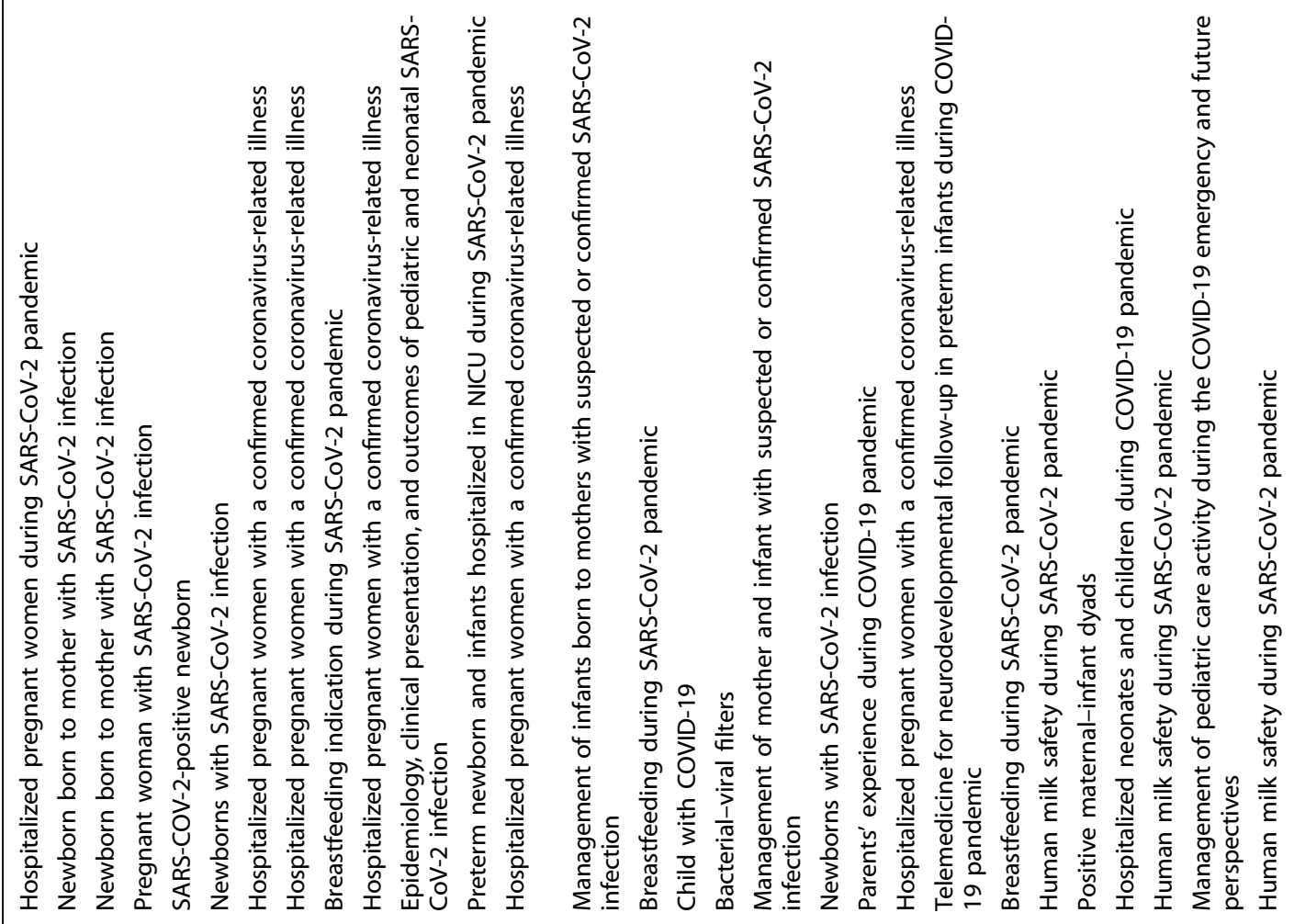

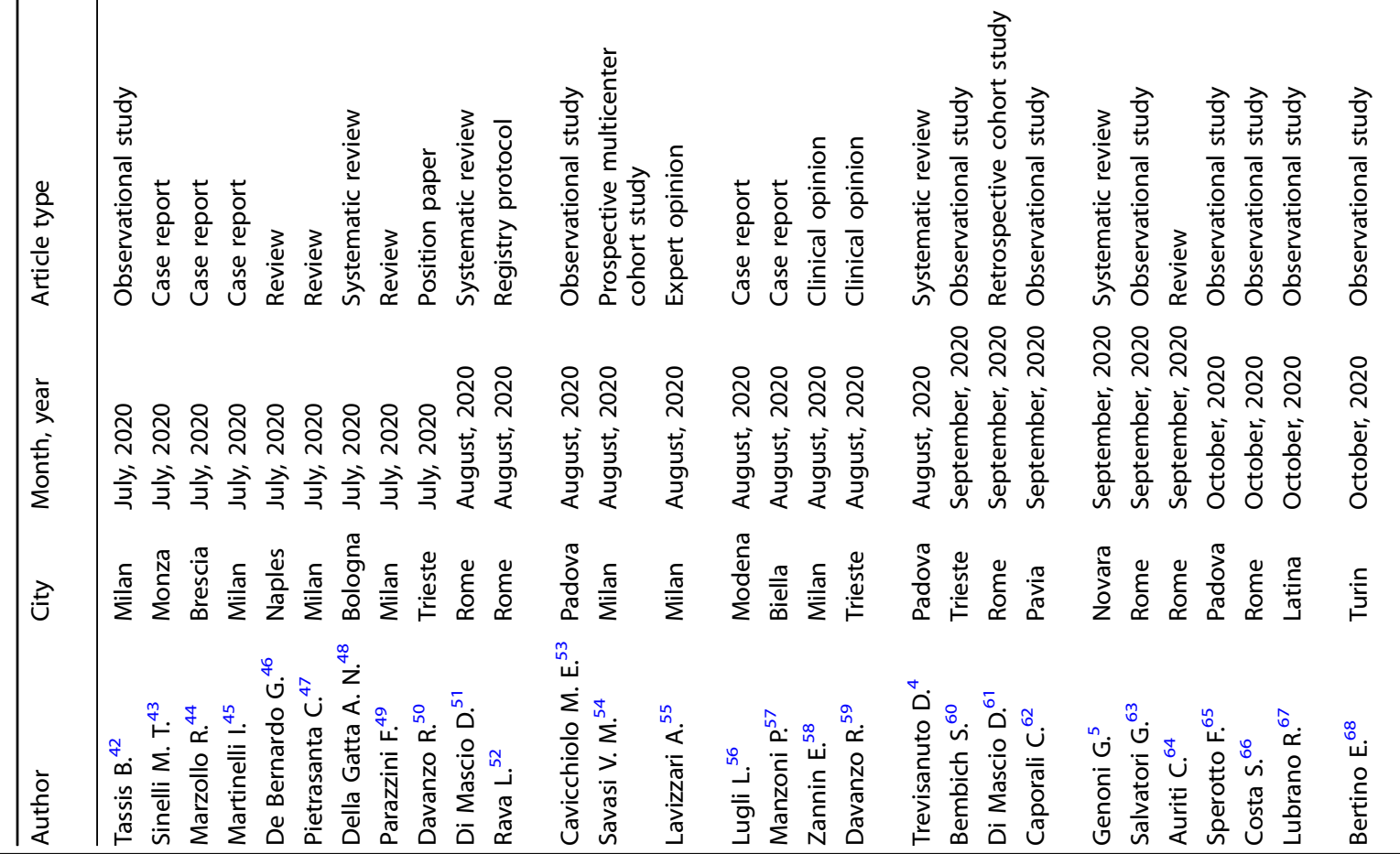


Italian hospitals. ${ }^{9,11,31,53}$ A woman's partner was allowed to attend the labor and delivery if his SARS-CoV-2 status was known but was not allowed to stay on the postpartum ward or it was permitted with time restrictions. ${ }^{37}$

Studies suggested concentrating COVID-19 cases, procedures, and medical devices in chosen third-level hub referral hospitals with a NICU. ${ }^{17}$

In case of delivery of a confirmed or suspected SARS-CoV-2 mother, the woman was assisted during delivery by a dedicated multidisciplinary team (obstetrician, gynecologist, anesthesiologist, neonatologist, neonatal nurse) in an isolation room and subsequently she was transferred to the maternity ward through a dedicated COVID-19 path, by obstetric staff donned with full personal protective equipment (PPE). ${ }^{17}$

There was no evidence to suggest the preferred mode of delivery, ${ }^{8,21}$ as vertical transmission rarely occurred, ${ }^{3,47,64}$ but studies recommended that suspected or positive woman deliveries should happen in a negative-pressure room, with the infant warmer at least at $2 \mathrm{~m}$ (6 feet) or in separate room. ${ }^{21}$

Finally, it was recommended to collect maternal specimens for SARS-CoV-2 testing (placenta, amniotic fluid) after delivery aiming to assess possible vertical transmissions. ${ }^{21,66}$

Management in the delivery room

In case of neonatal resuscitation, abovementioned studies $8,21,29$ suggested that it could be provided in an adjacent room or in the same room at least $2 \mathrm{~m}$ away from the mother with a physical barrier such as a curtain. All aerosol-generating procedures should be performed by using full PPE from the healthcare givers providing neonatal care (e.g., respirators, FFP2 or N95 masks, eye protection, gloves, and gowns). ${ }^{8,20,21,47}$

Delayed cord clamping could still be performed as a standard of care, while some author suggested avoiding maternal skin contact. ${ }^{21}$

Skin-to-skin contact was a conflicting topic. Some authors suggested a shared decision-making approach with the parents before the delivery regarding the potential risks and benefits of skin-to-skin care and kangaroo mother care, discussing the risks of exposure to both the neonate and healthcare providers, ${ }^{5}$ others did not recommend skin-to-skin contact. ${ }^{21,47}$ Davanzo et al. ${ }^{59}$ recommended skin-to-skin contact as far as mothers could carefully follow measures for infection prevention.

\section{Postnatal management}

The decision of the location of newborn's admittance, nursery vs. mother-baby unit vs. NICU was made based on gestational age, newborn's condition, need for antibiotics, intravenous fluids, respiratory support, post-delivery maternal conditions, and individual hospital policies. ${ }^{39}$ Most studies suggested a dedicated, specialized team caring for newborns born from COVID-19 mothers to minimize the transmission and spread of the SARSCoV-2 among healthcare workers. $8,21,53$

If the mother was positive for SARS-CoV-2, the infant should have been tested at or beyond 24 hours after birth. Separate swabs of the nasopharynx, oropharynx, and rectum were recommended depending on the availability and turnaround time of testing facilities. ${ }^{8,21}$

Screening at NICU admission was assessed by authors from Padova and Rome. ${ }^{31,32,53}$ Although the prevalence of COVID-19positive infants was low, both NICUs opted for a universal screening of newborns admitted to the Unit, their parents, and staff in order to avoid the spread of the virus in a high-risk ward. This policy allowed to identify early positive asymptomatic individuals, which were $2.2 \%$ of screened, ${ }^{53}$ and it has been demonstrated as the best approach to reduce SARS-CoV-2-related costs. $^{69}$ During the surveillance, no newborns tested positive for SARS-CoV-2. ${ }^{31,53}$ Positive neonates should be kept in negativepressure, isolated rooms, and when receiving respiratory support, the use of bacterial-viral filters would be recommended. ${ }^{8,58}$
Mother-baby dyad and breastfeeding management

Rooming in and breastfeeding were considered conflicting topics and changed over time.

Some authors initially suggested a different approach according to mother's COVID-19 status and clinical conditions. If the maternal COVID-19 status was negative or unknown or if a mother previously identified as COVID-19 positive or under investigation for COVID-19 was asymptomatic or paucisymptomatic at delivery, rooming breastfeeding was recommended. ${ }^{41,50}$ A recent study from Lombardy region strengthened the safety of rooming in and breastfeeding in positive women who can take care of their babies, demonstrating that only $1.6 \%$ of the enrolled newborns developed postnatal SARS-CoV-2 infection. ${ }^{70}$ On the contrary, when a mother with COVID-19 was too sick to care for the newborn, the neonate was suggested to be managed separately and fed with fresh expressed breast milk, with no need to pasteurize it, as human milk was not believed to be a vehicle of COVID-19. ${ }^{13}$

However, previous articles were more cautious about breastfeeding, in particular for feeding preterm infants admitted to the $\mathrm{NICU}^{21,31}$ suggesting only pasteurized human breast milk (donor or maternal) or formula milk. ${ }^{13}$

More recently, a case report, published by Lugli et al., and another Italian group described the presence of SARS-CoV-2 RNA in human milk samples. In both cases, no SARS-CoV-2 infection was documented in the babies who received the milk. ${ }^{56,66}$

Davanzo et al., in a letter, reinforced the concept of the importance of giving mother's milk to babies even in case of mother-child separation. ${ }^{40}$

The modality of milk donation, sanitization of milk container, and human milk bank reorganization during SARS-CoV-2 pandemic were also discussed suggesting testing all donor mothers for SARS-CoV-2 and sanitize milk containers using disinfectant wipes containing sodium hypochlorite and gloved hands. ${ }^{13}$

As SARS-CoV-2 is effectively inactivated by pasteurization at $62.5^{\circ} \mathrm{C}$ for $30 \mathrm{~min}$, universal screening of human milk bank samples and surfaces containers in order to detect SARS-CoV-2 has not been supported. ${ }^{63}$

Discharge criterion were also discussed: laboratory resolution of the infection (i.e., two negative nasopharyngeal swabs $24 \mathrm{~h}$ apart) was not considered a mandatory criterion for discharge. In case of positive mother or infant, provided good clinical conditions of both, the whole family is quarantined home. ${ }^{17}$ A strict follow-up of these families has been recommended. ${ }^{21}$

\section{NETS reorganization}

NETS is an essential service for centralizing SARS-CoV-2-positive or suspected newborns. During the pandemic period, it needed reorganization in terms of safety for the patients and the clinicians. Neonatal and pediatric working groups of the transport section of the European Society of Paediatric and Neonatal Intensive Care, the Neonatal Transport Study Group of the SIN, and a group of neonatologists of Veneto region published recommendations for this purpose. ${ }^{19,26,28}$ Use of individual protective N95 respirator (FFP3 mask), disposable water-repellent with long sleeves gown, double gloves, visor/goggles, disposable headgear, and disposable shoes has been recommended. The guidelines also indicated how to proceed to the disinfection of the transport incubator, the monitors, and ventilator with appropriate solutions, as well as all the equipment (i.e., masks, laryngoscope, selfinflating bag), which should be disposed or sterilized according to standard procedure. Guidelines also recommend storing protective wear equipment inside the ambulance in case of an unscheduled high risk for COVID-19 transport. Bellini et al. ${ }^{35}$ collected NETS data during the pandemic period and demonstrated that all the newly introduced procedures to perform a safe COVID-19 transport, in particular dressing and undressing procedures, doubled the total transport time. 


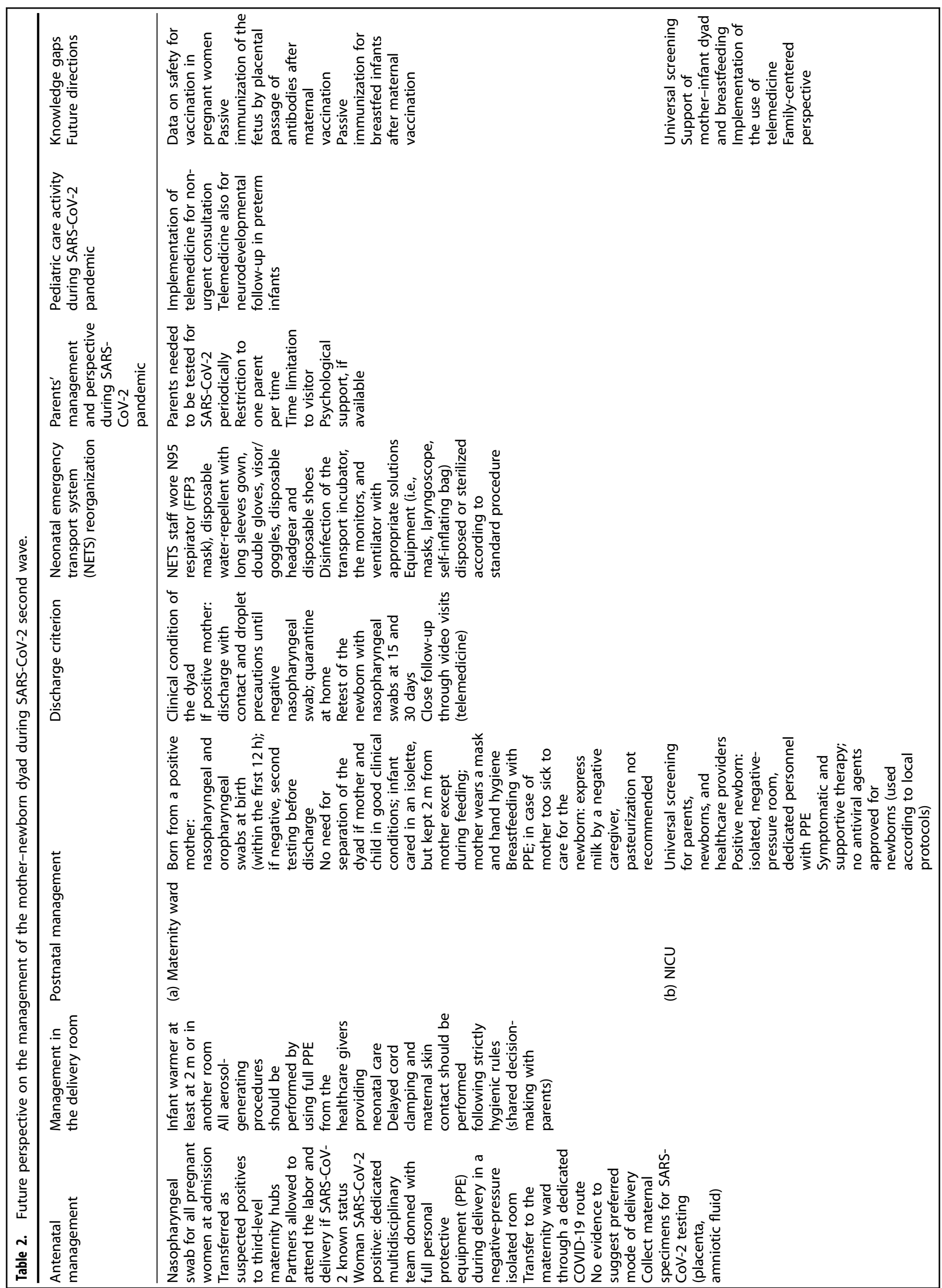


Parents' management and perspective during SARS-CoV-2 pandemic

Parents were allowed to visit their babies with some restrictions: they should have been periodically tested for SARS-CoV-2 through nasopharyngeal swabs with a negative result; furthermore, the use of PPE was mandatory.

Two articles analyzed parents' perspective during COVID-19 pandemic. Lista and Bresesti ${ }^{18}$ expressed his opinion as neonatologist who faced the problem of fatherhood during SARS-CoV-2. The author underlined the importance to give more active support to new families by all the healthcare providers involved with birth. Bembich et al. conducted an interview to parents of infants hospitalized in NICU about COVID-19 visitor restriction reporting that most parents expressed dysphoric emotions and relational suffering, indicating that it was very difficult for them to deal with such policies. ${ }^{60}$

\section{Future perspectives}

In the pandemic period, telemedicine was implemented to perform non-urgent consultation and for neurodevelopmental follow-up in preterm infants, with a positive feedback from healthcare providers and parents. ${ }^{34,36,38}$

Table 2 summarized the key points for managing the second wave of SARS-CoV-2 pandemic.

\section{DISCUSSION}

The COVID-19 pandemic has put under pressure all the national health systems across Europe, but unfortunately, there has been a poor coordination between countries in the management of these SARS-CoV-2 outbreaks. ${ }^{71}$

This review summarizes the available evidence from neonatal COVID-19 management in Italy analyzing all the published paper in this specific field of interest. Most of the retrieved papers were clinical opinion or position paper, which means that the majority of recommendations were not evidence based.

Despite this paucity of information about SARS-CoV-2 at the beginning of the pandemic and the absence of consensus guidelines, our review shows that most of the Italian maternity and neonatal wards adopted similar approaches and they optimize patients' management overtime. Our results demonstrate a less restrictive testing model in the papers published at the beginning of the pandemic, when most centers tested only symptomatic pregnant women for SARS-CoV-2 with a change to a screening policy of all pregnant women overtime. ${ }^{72}$ On the other hand, the management of newborns of infected or suspected mothers was initially more restrictive in some hospitals: these neonates were placed in isolation rooms based on the available literature at that time and they were fed by formula milk, ${ }^{8,21}$ separation of the dyad was recommended for all the hospitalizations based on Chinese guidelines, the only country who experienced COVID-19 before the arrival of the infection in Italy in February 2020. ${ }^{72}$ However, this full restrictive approach was not supported by the official guidelines of the SIN that continued to recommend both skin-to-skin contact and breastfeeding with hygienic measures since beginning of the pandemic according to the World Health Organization and UNICEF recommendations. ${ }^{6}$ In the following months, when positive asymptomatic cases dramatically increased, Italian maternity wards opted for a universal screening approach, testing all pregnant women admitted for delivery, their newborns, and their partners. This approach detected a $2.2 \%$ of positive individuals, who were asymptomatic, and likely contributed to avoid a spread of the virus in high-risk wards, such as NICUs. ${ }^{53}$ Nowadays, universal screening approach could be considered an even more precious approach as new variants, which seem more contagious for children and younger people, are spreading through Europe. ${ }^{73}$

Separation of the dyad occurred only if the mother was too sick for caring for the baby or when the infants needed intensive care.
Mothers were encouraged to provide breastfeeding while rooming-in, following appropriate isolation precautions based on the Centers for Disease Control and Prevention guidance. ${ }^{74}$ Skin-toskin care was allowed with PPE and hygienic measures. Healthy asymptomatic newborns were generally discharged early and left to a healthy asymptomatic caregiver. Systematic reviews and meta-analysis demonstrated that mother-neonate rooming-in was associated with a higher incidence of SARS-CoV-2 infections, ${ }^{3}$ but approximately half of the infected neonates were asymptomatic, and if they developed COVID-19, outcome was generally favorable. , $^{3,61}$

Breastfeeding was a conflicted topic. There were few case reports demonstrating SARS-CoV-2 RNA presence in nonpasteurized human milk, but no evidence of infection in newborn fed with positive milk, suggesting a possible protective role of the immunoglobulins included in the human milk. ${ }^{56,66,68}$

A recent systematic review of all the published guidelines highlighted that all documents recommend breastfeeding or feeding with expressed maternal milk with the only exception for the Chinese recommendations. ${ }^{5}$ In the setting of COVID-19, studies demonstrated that separation of mother-newborn dyads impacts breastfeeding outcomes, with lower rates of breastfeeding both during hospitalization and at home., 5 The SIN promoted breastfeeding during SARS-CoV-2 pandemic, even in positive symptomatic women, following strictly hygienic rules. ${ }^{6}$

Despite the importance, psychological and emotional aspects caused by SARS-CoV-2 restriction in maternity wards and in NICUs were studied only in two papers. ${ }^{18,60}$

Telemedicine was considered a useful tool for non-urgent follow-up consultation both from healthcare providers and parents and needed to be implemented. ${ }^{62,67}$

Current evidences are missing on the safety and efficacy of vaccination against SARS-CoV-2 of pregnant and lactating women. Even though these categories were not included in the development and clinical evaluation of COVID-19 vaccines in the premarket phase, the Italian Health Institute ${ }^{76}$ and SIN, ${ }^{77}$ in line with most of the international societies and agencies, ${ }^{78-80}$ suggested vaccination for both groups when at high risk of exposure to SARS-CoV-2 or who have comorbidities, which can lead to a more severe disease. Regarding the timing of the vaccination, since no data were available on its safety during pregnancy, the Italian Health Institute suggested delaying it in the second trimester, to avoid situations at increased risk for the fetus in the first trimester (e.g., neural tube defects related to fever or miscarriage). ${ }^{76} \mathrm{~A}$ recent preprint paper describes the first case of anti-SARS-CoV-2 antibodies detected in the cord blood of a neonate born to a mother immunized with the first dose of an mRNA COVID-19 vaccine 3 weeks earlier. A burning question is to evaluate on larger cohorts the protection of the newborn by placental passage of antibodies as for influenza and pertussis vaccination. ${ }^{81}$

Our hope in the next few months lies in the possibility of sharing the acquired knowledge on a larger scale in a European effort in order to fight this pandemic as rapidly and decisively as possible.

\section{CONCLUSION}

There was a paucity of quality of data regarding the management of mother and neonates during SARS-CoV-2 pandemic. Nevertheless, our review shows a change toward universal screening, support of safe mother-infant dyad and breastfeeding, implementation of the use of telemedicine, and a family-centered perspective. Evidence and suggestions evolved over the pandemic period and the current review can be useful in the management of the mother-neonate dyad during the SARS-CoV-2 second wave. National scientific societies, such as the SIN, have played a central role in coordinating and guiding every-day clinical practices in this challenging time. Italian neonatologists have played an active role 
in producing official guidelines and reporting data that have contributed to improve the care of neonates. A joint European action plan is mandatory to face COVID-19 with more awareness.

\section{AUTHOR CONTRIBUTIONS}

M.E.C. wrote the initial draft and revised the literature and approved the final manuscript as submitted. D.T. contributed to write the draft and revised the literature and approved the final manuscript as submitted. E.P. and L.M. critically reviewed the manuscript. F.M. provided relevant expertize and critically reviewed the manuscript. E.B. contributed to the study concept, study design, and writing of the manuscript and critically reviewed the manuscript. All authors approved the final manuscript as submitted and agree to be accountable for all aspects of the work.

\section{ADDITIONAL INFORMATION}

Competing interests: The authors declare no competing interests.

Patient consent: Patient consent was not required

Publisher's note Springer Nature remains neutral with regard to jurisdictional claims in published maps and institutional affiliations.

\section{REFERENCES}

1. WHO. Coronavirus disease (COVID-19) weekly epidemiological update and weekly operational update. https://www.who.int/emergencies/diseases/novelcoronavirus-2019/situation-reports (2020).

2. Dong, Y. et al. Epidemiology of COVID-19 among children in China. Pediatrics 145, e20200702 (2020).

3. Raschetti, R. et al. Synthesis and systematic review of reported neonatal SARSCoV-2 infections. Nat. Commun. 11, 5164 (2020).

4. Trevisanuto, D. et al. Coronavirus infection in neonates: a systematic review. Arch. Dis. Child. Fetal Neonatal Ed. https://doi.org/10.1136/archdischild-2020-319837 (2021).

5. Genoni, G. et al. Management and nutrition of neonates during the COVID-19 pandemic: a review of the existing guidelines and recommendations. Am. J. Perinatol. 37, S46-S53 (2020).

6. Società Italiana di Neonatologia. Allattamento e gestione del neonato in corso di pandemia da SARS-COV-2. Versione 3, 10 maggio 2020. https://www.sinneonatologia.it/ (2020)

7. Liberati, A. et al. The PRISMA statement for reporting systematic reviews and meta-analyses of studies that evaluate healthcare interventions: explanation and elaboration. BMJ 339, b2700 (2009).

8. Chandrasekharan, $P$. et al. Neonatal resuscitation and postresuscitation care of infants born to mothers with suspected or confirmed SARS-CoV-2 infection. Am. J. Perinatol. 37, 813-824 (2020).

9. Di Mascio, D. et al. Outcome of Coronavirus spectrum infections (SARS, MERS, COVID 1 -19) during pregnancy: a systematic review and meta-analysis. Am. J. Obstet. Gynecol. 2, 100107 (2020).

10. De Rose, D. U. et al. Novel coronavirus disease (COVID-19) in newborns and infants: what we know so far. Ital. J. Pediatr. 46, 56 (2020).

11. Ferrazzi, E. et al. Vaginal delivery in SARS-CoV-2-infected pregnant women in Northern Italy: a retrospective analysis. BJOG https://doi.org/10.1111/14710528.16278 (2020).

12. Canarutto, D. et al. COVID-19 infection in a paucisymptomatic infant: raising the index of suspicion in epidemic settings. Pediatr. Pulmonol. 55, E4-E5 (2020).

13. De Rose, D. U. et al. Use of disinfectant wipes to sanitize milk's containers of human milk bank during COVID-19 pandemic. J. Hum. Lact. 36, 547-549 (2020).

14. Salvatori, G. et al. Managing COVID-19-positive maternal-infant dyads: an Italian experience. Breastfeed. Med. 15, 347-348 (2020).

15. Perrone, S. et al. Lack of viral transmission to preterm newborn from a COVID-19 positive breastfeeding mother at 11 days postpartum. J. Med. Virol. https://doi. org/10.1002/jmv.26037 (2020).

16. Perrone, $\mathrm{S}$. et al. Report of a series of healthy term newborns from convalescent mothers with COVID-19. Acta Biomed. 91, 251-255 (2020).

17. Pietrasanta, C. et al. Management of the mother-infant dyad with suspected or confirmed SARS-CoV-2 infection in a highly epidemic context. J. Neonatal Perinatal Med. 13, 307-311 (2020).

18. Lista, G. \& Bresesti, I. Fatherhood during the COVID-19 pandemic: an unexpected turnaround. Early Hum. Dev. 144, 105048 (2020).

19. Cavicchiolo, M. E. et al. Neonatal emergency transport system during COVID-19 pandemic in the Veneto Region: proposal for standard operating procedures. Pediatr. Res. 89, 399-401 (2020).
20. Shalish, W., Lakshminrusimha, S., Manzoni, P., Keszler, M. \& Sant'Anna, G. M. COVID-19 and neonatal respiratory care: current evidence and practical approach. Am. J. Perinatol. 37, 780-791 (2020).

21. Trevisanuto, D. et al. Neonatal resuscitation where the mother has a suspected or confirmed novel coronavirus (SARS-CoV-2) infection: suggestion for a pragmatic action plan. Neonatology 117, 133-140 (2020).

22. Liguoro, I. et al. SARS-COV-2 infection in children and newborns: a systematic review. Eur. J. Pediatr. 179, 1029-1046 (2020)

23. Procianoy, R. S., Silveira, R. C., Manzoni, P. \& Sant'Anna, G. Neonatal COVID-19: little evidence and the need for more information. J. Pediatr. 96, 269-272 (2020).

24. Buonsenso, D. et al. Neonatal late onset infection with severe acute respiratory syndrome coronavirus 2. Am. J. Perinatol. 37, 869-872 (2020).

25. Saccone, G., Carbone, F. I. \& Zullo, F. The novel coronavirus (2019-nCoV) in pregnancy: what we need to know. Eur. J. Obstet. Gynecol. Reprod. Biol. 249, 92-93 (2020).

26. Terheggen, $U$. et al. European consensus recommendations for neonatal and paediatric retrievals of positive or suspected COVID-19 patients. Pediatr. Res. https://doi.org/10.1038/s41390-020-1050-z (2020).

27. Lavizzari, A. et al. International comparison of guidelines for managing neonates at the early phase of the SARS-CoV-2 pandemic. Pediatr. Res. https://doi.org/ 10.1038/s41390-020-0976-5 (2020).

28. Bellini, C. \& Gente, M. Neonatal transport and COVID-19 outbreak. Air Med. J. 39, 154-155 (2020).

29. Trevisanuto, D. et al. Management of mothers and neonates in low-resources setting during Covid-19 pandemia. J. Matern. Fetal Neonatal Med. https://doi.org/ 10.1080/14767058.2020.1784873 (2020).

30. Carosso, A. et al. Pre-labor anorectal swab for SARS-CoV-2 in COVID-19 pregnant patients: is it time to think about it? Eur. J. Obstet. Gynecol. Reprod. Biol. 249, 98-99 (2020).

31. Cavicchiolo, M. E., Lolli, E., Trevisanuto, D. \& Baraldi, E. Managing a tertiary-level NICU in the time of COVID-19: lessons learned from a high-risk zone. Pediatr. Pulmonol. 55, 1308-1310 (2020).

32. De Rose, D. U. et al. Reshaping neonatal intensive care units (NICUs) to avoid the spread of severe acute respiratory coronavirus virus 2 (SARS-CoV-2) to high-risk infants. Infect. Control Hosp. Epidemiol. https://doi.org/10.1017/ice.2020.310 (2020).

33. Morniroli, D. et al. Human sialome and coronavirus disease-2019 (COVID-19) pandemic: an understated correlation? Front. Immunol. 11, 1480 (2020).

34. Marino, S., Ruggieri, M. \& Falsaperla, R. Is SARSCoV-2 nasopharyngeal swab still a gold standard in children? Med. Hypotheses 144, 110041 (2020).

35. Bellini, C. COVID-19 outbreak impact on neonatal emergency transport. Pediatr. Res. https://doi.org/10.1038/s41390-020-1027-y (2020).

36. Ferrazzi, E. M. et al. COVID-19 Obstetrics Task Force, Lombardy, Italy: executive management summary and short report of outcome. Int. J. Gynaecol. Obstet. 149, 377-378 (2020)

37. Trippella, G. et al. COVID-19 in pregnant women and neonates: a systematic review of the literature with quality assessment of the studies. Pathogens 9, 485 (2020).

38. Manzoni, P. et al. Impact of COVID-19 epidemics in paediatric morbidity and utilisation of Hospital Paediatric Services in Italy. Acta Paediatr. https://doi.org/ 10.1111/apa.15435. (2020)

39. Procianoy, R. S., Silveira, R. C., Manzoni, P. \& Sant'Anna, G. Neonatal COVID-19: little evidence and the need for more information. J. Pediatr. 96, 269-272 (2020).

40. Davanzo, R. Breast feeding at the time of COVID-19: do not forget expressed mother's milk, please. Arch. Dis. Child. Fetal Neonatal Ed. 105, 455 (2020).

41. Buonsenso, D. et al. Clinical role of lung ultrasound for diagnosis and monitoring of COVID-19 pneumonia in pregnant women. Ultrasound Obstet. Gynecol. 56, 106-109 (2020).

42. Tassis, B. et al. Effectiveness of a COVID-19 screening questionnaire for pregnant women at admission to an obstetric unit in Milan. Int. J. Gynaecol. Obstet. 150, 124-126 (2020).

43. Sinelli, M. et al. Early neonatal SARS-CoV-2 infection manifesting with hypoxemia requiring respiratory support. Pediatrics 146, e20201121 (2020).

44. Marzollo, R. et al. Possible coronavirus disease 2019 pandemic and pregnancy: vertical transmission is not excluded. Pediatr. Infect. Dis. J. 39, e261-e262 (2020).

45. Martinelli, I. et al. Pulmonary embolism in a young pregnant woman with COVID19. Thromb. Res. 191, 36-37 (2020).

46. De Bernardo, G. et al. The clinical course of SARS-CoV-2 positive neonates. J. Perinatol. 40, 1462-1469 (2020).

47. Pietrasanta, C. et al. SARS-CoV-2 infection and neonates: a review of evidence and unresolved questions. Pediatr. Allergy Immunol. 31, 79-81 (2020).

48. Della Gatta, A. N. et al. Coronavirus disease 2019 during pregnancy: a systematic review of reported cases. Am. J. Obstet. Gynecol. 223, 36-41 (2020).

49. Parazzini, F. et al. Delivery in pregnant women infected with SARS-CoV-2: a fast review. Int. J. Gynaecol. Obstet. 150, 41-46 (2020). 
Italian neonatologists and SARS-CoV-2: lessons learned to face coming new... ME Cavicchiolo et al.

50. Davanzo, R. et al. Breastfeeding and coronavirus disease-2019: ad interim indications of the Italian Society of Neonatology endorsed by the Union of European Neonatal \& Perinatal Societies. Matern. Child Nutr. 16, e13010 (2020).

51. Huntley, B. J. F. et al. Rates of maternal and perinatal mortality and vertical transmission in pregnancies complicated by severe acute respiratory syndrome coronavirus 2 (SARS-Co-V-2) infection: a systematic review. Obstet. Gynecol. 136, 303-312 (2020).

52. De Luca, D. et al. The EPICENTRE (ESPNIC Covid pEdiatric Neonatal Registry) initiative: background and protocol for the international SARS-CoV-2 infections registry. Eur. J. Pediatr. 179, 1271-1278 (2020).

53. Cavicchiolo, M. E. et al. Universal screening of high-risk neonates, parents, and staff at a neonatal intensive care unit during the SARS-CoV-2 pandemic. Eur. J. Pediatr. https://doi.org/10.1007/s00431-020-03765-7 (2020).

54. Savasi, V. M. et al. Clinical findings and disease severity in hospitalized pregnant women with coronavirus disease 2019 (COVID-19). Obstet. Gynecol. 136, 252-258 (2020).

55. Molloy, E. J. et al. Neonates in the COVID-19 pandemic. Pediatr. Res. https://doi. org/10.1038/s41390-020-1096-y (2020).

56. Lugli, L. et al. An uninfected preterm newborn inadvertently fed SARS-CoV-2positive breast milk. Pediatrics 146, e2020004960 (2020).

57. Manzoni, P. et al. Uncommon presentation of coronavirus disease 2019 infection in a child. Pediatr. Infect. Dis. J. 39, e212-e213 (2020).

58. Zannin, E. et al. Bacterial-viral filters to limit the spread of aerosolized respiratory pathogens during neonatal respiratory support in a pandemic era. Pediatr. Res. https://doi.org/10.1038/s41390-020-1102-4 (2020).

59. Davanzo, R., Merewood, A. \& Manzoni, P. Skin-to-skin contact at birth in the COVID-19 era: in need of help! Am. J. Perinatol. 37, S1-S4 (2020).

60. Bembich, S. et al. Parents experiencing NICU visit restrictions due to COVID-19 pandemic. Acta Paediatr. 110, 940-941 (2020).

61. WAPM (World Association of Perinatal Medicine) Working Group on COVID-19. Maternal and perinatal outcomes of pregnant women with SARS-CoV-2 infection. Ultrasound Obstet. Gynecol. 57, 232-241 (2021).

62. Caporali, C. et al. Challenges and opportunities for early intervention and neurodevelopmental follow-up in preterm infants during COVID-19 pandemic. Child Care Health Dev. 47, 140-141 (2020).

63. Salvatori, G. et al. Universal screening for SARS-CoV-2 of all human milk bank samples. J. Hum. Lact. 37, 40-42 (2020).

64. Auriti, C. et al. Vertical transmission of SARS-CoV-2 (COVID-19): are hypotheses more than evidences? Am. J. Perinatol. 37, S31-S38 (2020).

65. Sperotto, F. et al. Unplanned and medical admissions to pediatric intensive care units significantly decreased during COVID-19 outbreak in Northern Italy. Eur. J. Pediatr. 180, 643-648 (2020).
66. Costa, S. et al. Excretion of SARS-CoV-2 in human breast milk. Clin. Microbiol. Infect. 26, 1430-1432 (2020).

67. Lubrano, R. et al. Point of view of the Italians pediatric scientific societies about the pediatric care during the COVID-19 lockdown: what has changed and future prospects for restarting. Ital. J. Pediatr. 46, 142 (2020). and follow-up of their infants. Front. Pediatr. 8, 597699 (2020).

69. Galderisi, A. et al. The aftermath of SARS-CoV-2 in NICU: saving or checking accounts? Projected cost-effectiveness analysis. Eur. J. Pediatr. https://doi.org/ 10.1007/s00431-020-03884-1 (2021).

70. Ronchi, A. et al. Evaluation of rooming-in practice for neonates born to mothers with severe acute respiratory syndrome coronavirus 2 infection in Italy. JAMA Pediatr. 175, 260-266 (2021).

71. Horton, R. Offline: Europe and COVID-19-struggling with tragedy. Lancet 396, 1713 (2020). agement for the prevention and control of the 2019 novel coronavirus infection (first edition). Ann. Transl. Med. 8, 47 (2020).

73. Brookman, S. et al. Effect of the new SARS-CoV-2 variant B.1.1.7 on children and young people. Lancet Child Adolesc. Health 5, e9-e10 (2021).

74. CDC. Coronavirus disease (COVID-19) and breastfeeding. https://www.cdc.gov/ breastfeeding/breastfeeding-special-circumstances/maternal-or-infant-illnesses/ covid-19-and-breastfeeding.html (2020).

75. Verma, S. et al. Outcomes of maternal- newborn dyads after maternal SARS-CoV2. Pediatrics 146, e2020005637 (2020).

76. Istituto Superiore di Sanità. Indicazioni ad interim per gravidanza, parto, allattamento e cura dei piccolissimi 0-2 anni in risposta all'emergenza COVID-19. https://www.iss.it (2021).

77. Società Italiana di Neonatologia. Indicazioni ad interim su Vaccinazione contro il COVID - 19 in gravidanza e allattamento. https://www.sin-neonatologia.it/indicazioniad-interim-su-vaccinazione-contro-il-covid-19-in-gravidanza-e-allattamento/ (2021).

78. CDC. Vaccine recommendations and guidelines of the Advisory Committee on Immunization Practices (ACIP). https://www.cdc.gov/vaccines/hcp/acip-recs/vaccspecific/covid-19/clinicalconsiderations.html (2021).

79. American College of Obstetricians and Gynecologists (ACOG). Vaccinating pregnant and lactating patients against COVID-19. https://www.acog.org/clinical/ clinicalguidance/practiceadvisory/articles/2020/12/vaccinating-pregnant-andlactating-patients-against-covid-19 (2021).

80. Academy of Breastfeeding Medicine. Considerations for COVID-19 vaccination in lactation. https://www.bfmed.org/abm-statement-considerations-for-covid-19vaccination-in-lactation (2021).

81. Paul, G. \& Chad, R. Newborn antibodies to SARS-CoV-2 detected in cord blood aftermaternal vaccination - a case report. BMC Pediatr. 2021;21:138.
68. Bertino, E. et al. Detection of SARS-CoV-2 in milk from COVID-19 positive mothers

72. Wang, L. et al. Chinese expert consensus on the perinatal and neonatal man- 